\title{
Discussion on Instrument and Equipment Management of Scientific Research Laboratory
}

Dan Zhao

Institute of Agro-Bioengineering, Guizhou University, Guiyang 550025, Guizhou, China.

Funding Project: This research was funded by the Guizhou Provincial Department of Education "Biology Postgraduate Excellent Talents Training"project

Abstract: With the continuous investment of scientific research funds, the hardware conditions of many scientific research laboratories in Colleges and universities have been widely constructed and developed. The share of instruments in scientific research laboratories has been increasing, and the problems in the management of instruments have gradually emerged. Therefore, more and more colleges and universities pay attention to the maximum benefit of scientific research instruments and equipment and the importance of discussing the management of instruments and equipment. This paper discusses and analyzes the problems existing in the management of instruments and equipment in the scientific research laboratory, and puts forward the problems and solutions to improve the management of experimental instruments and equipment in Colleges and universities in the new era.

Keywords: Laboratory; Instruments and Equipment; Management

Instruments and equipment are necessary tools for colleges and universities to carry out research work, especially laboratories based on scientific research, which are often used as one of the important indicators to measure the comprehensive strength of laboratories. However, in many laboratories, there is the problem of "purchasing, neglecting management", leading to idle large-scale instruments, frequent damage to conventional instruments, delayed maintenance, and a large number of instruments becoming scrap. Therefore, how to maximize the benefits of scientific research instruments, realize the standardization and scientific management of scientific research laboratory instruments and equipment, so that they can serve scientific research to the greatest extent. Therefore, we will discuss the problems and countermeasures in the instrument management of the key laboratory of the Ministry of Education.

\section{Problems in the management of experimental equipment}

\subsection{Large-scale equipment is idle and the utilization rate is low}

The laboratory has a series of high-precision instruments such as scanning electron microscope, transmission electron microscope, con focal microscope, flow cytometer, fluorescent quantitative PCR instrument, digital PCR instrument, and gas/liquid mass spectrometry, with a total value of more than 20 million yuan However, the utilization rate of most large instruments is low, and the maintenance and repair costs are high, resulting in most of the instruments not being used badly, but broken. There are two main reasons for the analysis: one is the high cost of using the instrument, such as a DNA sequencer, which costs about 6000-7000 for a kit, and needs to be operated and analyzed by yourself. However, when sent to the company, a base is only 1 yuan, and it will be available within three days. Results can be obtained; second, there is a lack of professional

Copyright(C) 2020 Dan Zhao

doi: 10.18686/ahe.v4i10.2949

This is an open-access article distributed under the terms of the Creative Commons Attribution Non-Commercial License (http://creativecommons. org/licenses/by-nc/4.0/), which permits unrestricted non-commercial use, distribution, and reproduction in any medium, provided the original work is properly cited. 
instrument management personnel. Most of the teachers in the laboratory are teachers, and they undertake the school's teaching and research work. Each time you need to use the instrument, you must relearn the operation of the instrument. This is slow and costly. More time, so teachers would rather spend money to let the company do it. In addition, the utilization rate of the instrument and whether there is a full-time instrument administrator should be considered when the instrument purchase demonstration. The instrument ultimately needs professional and technical personnel to operate the instrument. Most of the reason why the instrument is idle is that no one will operate the instrument. There should be someone first and then the instrument, instead of waiting for the instrument to arrive before arranging someone to learn it. The consequence of this is that the person who arranged the learner will not use it, and will soon forget how to use it.

\subsection{Conventional instruments are frequently used and damaged frequently}

Compared with large-scale precision instruments, conventional instruments are more commonly used instruments in scientific research laboratories, such as shakers, incubators, and water purifiers, which operate 24 hours a day. In scientific research laboratories, subject groups are the basic units. Each subject group includes doctoral students, master students, and a small number of undergraduates. There are many users, uneven quality, and lack of responsibility, which can easily cause instrument damage. For example, small centrifuges often cause bearing damage due to unbalanced centrifuge tubes, and maintenance costs can range from hundreds to thousands.

\subsection{Lack of full-time instrument management personnel and chaotic instrument management}

In scientific research laboratories, its members include teacher series and experiment series. The teacher series generally believe that teaching and scientific research are their own jobs, and laboratory management and instrument management are part of the experimental series. The experimental technicians not only have to undertake laboratory management work, but also complete certain teaching and research work, and the number of instruments has far exceeded the number of experimental technicians, often one experimental technician has to manage more than 10 large-scale The instrument is also responsible for the management of a large number of conventional instruments. The consequence is that all instrument management is floating on the surface, and instrument management can only be handled.

\subsection{The rules and regulations are well-organized and the enforcement is insufficient}

The laboratory has formulated the "Management Measures for Large Precision Instruments and Equipment", "Laboratory Instrument Manager Job Responsibilities and Assistant Management Responsibilities", and has compiled "Operational Procedures and Precautions" for each large instrument exceeding 200, 000. However, due to There is no reward and punishment system, at the same time there is a lack of full-time management personnel, and insufficient supervision of the equipment, resulting in a lack of responsibility for the assistant management and users, and it is not related to their own high hangings, resulting in the failure to implement the rules and regulations, and become a dead letter.

\subsection{There is no special maintenance fund for the instrument, making maintenance difficult}

At present, the maintenance of the instrument mainly depends on the project funds of the scientific research personnel. For example, the maintenance cost of the filter column of the pure water instrument is close to 20, 000 yuan a year. This does not include the maintenance cost of the instrument failure, and the maintenance cost of the scientific research personnel is limited. Schools generally apply for repairs of large instruments once a year, and the procedures are complicated. Generally, the period from reporting to completion of repairs is more than one year. Reporting and repairing of conventional instruments is also difficult and the cycle is long, which often results in damage to the instrument that cannot be repaired.

\section{Measures to strengthen the management of laboratory equipment}

\subsection{Do a good job in large-scale instrument sharing services and build a team of full-time instrument management personnel}

Relying on the large-scale instrument sharing platform established by the school, scientific research laboratory instruments are shared inside and outside the school. Through the platform, let the instrument work, improve the efficiency of the use of the instrument, and promote the continuous learning of the instrument management personnel, so that the instrument sharing service can be better provided, and the paid use also improves the enthusiasm of the instrument management personnel. Strengthen and attach importance to the training of laboratory technicians, regularly arrange for laboratory technicians to go 
out to learn advanced laboratory management experience and training in the use of large instruments. At the same time, fulltime instrument management personnel should be assigned according to the total value of the instrument.

\subsection{Improve the instrument management system and carry out training on the use of instruments from time to time}

The main body of the instrument use enables students to formulate matching reward and punishment measures, which can stimulate students' management enthusiasm, restrain students from taking good care of the instrument, and ensure the normal operation of the laboratory. The instrument management can be linked to the student assistance management, and a certain subsidy will be given; and for students who fail to comply with the rules and regulations and have not changed after repeated teaching, appropriate punishments will be given to stop the experiment for more than one week before entering the laboratory to carry out the experiment.

In addition, the laboratory should organize management personnel to carry out training on the use of instruments from time to time. Both large-scale and conventional instruments should be strengthened in the use of training. This can increase the use rate of the instrument, and often sound the alarm to avoid improper operation. Damage can affect the development of the experiment. Regular instrument management briefings are held to report long-term non-compliance with laboratory instrument management systems or recent problems in instrument management and use. The alarm bell strikes so that students can keep the instrument use and precautions in mind.

\subsection{The laboratory adopts access control management and strictly implements the registration system for instrument use}

The big data management mode is adopted, and each room is equipped with a smart door lock. The management personnel set permissions for the room according to the specific conditions of the instrument. Only the management personnel and assistant management personnel have the right to open large instruments. Commonly used instruments authorize every person who uses the instrument., Managers can monitor every person who enters and exits the room in the background to achieve clear accountability and strengthen the controllability of management. At the same time, the equipment registration system is strictly implemented, and those who do not register without reason will be held accountable according to the records of entering and leaving the room (such as stopping the room for a week).

\subsection{Establish an instrument maintenance and repair fund}

With the continuous development of laboratories and the increasing number of teachers in scientific research posts, they will inevitably face many funding problems. Instruments are particularly prominent. On the one hand, they apply to schools for special funds for instrument maintenance. On the other hand, to strengthen teachers' sense of ownership and at the same time It also speeds up the instrument maintenance cycle, sets up an internal instrument maintenance fund in the laboratory, and draws a certain percentage of the maintenance fund based on the approved amount of the project declared by the teachers for small sporadic maintenance in the laboratory.

In short, laboratory instrument management is complicated, chaotic, and numerous. It requires the joint efforts and care of laboratory teachers and classmates to gradually improve it. Instrument management is linked to each other to ensure that the normal operation of the laboratory is not affected.

\section{References}

1. Tang S, Liu K, Xiao K. Research on the existing problems and countermeasures of university laboratory instrument and equipment Management. Science and Technology Economy Market 2012; (09): 62-64.

2. Ouyang K, Que Q, Huang X. Problems and countermeasures in the management of college-level experimental equipment and equipment. Education and Teaching Forum 2019; 12: 9-10

3. Zhang L, Chen D, Lian Y, et al. Current situation and thinking of equipment management in local undergraduate colleges. Guangdong Chemical Industry 2019; 8: 225-227

4. Chen L. The status quo and analysis of large-scale equipment management in colleges and universities. Management Observation 2018; (05): 106-107.

5. Meng T, Cheng J, Zhang R. Exploration of the use and management of large-scale equipment in key laboratories. Guangdong Chemical Industry 2018; 45(21): 66+55. 